\title{
On a Pointwise Convergence of Quasi-Periodic-Rational Trigonometric Interpolation
}

\author{
Arnak Poghosyan and Lusine Poghosyan \\ Institute of Mathematics of National Academy of Sciences of Armenia, Bagramian Avenue 24/5, 0019 Yerevan, Armenia \\ Correspondence should be addressed to Arnak Poghosyan; arnak@instmath.sci.am
}

Received 26 November 2013; Accepted 7 March 2014; Published 3 April 2014

Academic Editor: Nicolas Crouseilles

Copyright ( 2014 A. Poghosyan and L. Poghosyan. This is an open access article distributed under the Creative Commons Attribution License, which permits unrestricted use, distribution, and reproduction in any medium, provided the original work is properly cited.

\begin{abstract}
We introduce a procedure for convergence acceleration of the quasi-periodic trigonometric interpolation by application of rational corrections which leads to quasi-periodic-rational trigonometric interpolation. Rational corrections contain unknown parameters whose determination is important for realization of interpolation. We investigate the pointwise convergence of the resultant interpolation for special choice of the unknown parameters and derive the exact constants of the main terms of asymptotic errors.
\end{abstract}

\section{Introduction}

The quasi-periodic (QP) interpolation $I_{N, m}(f, x), m \geq 0$ ( $m$ is integer) and $x \in[-1,1]$, interpolates function $f$ on equidistant grid

$$
x_{k}=\frac{k}{N}, \quad|k| \leq N
$$

and is exact for a quasi-periodic function

$$
e^{i \pi n \sigma x}, \quad|n| \leq N, \quad \sigma=\frac{2 N}{2 N+m+1}
$$

with period $2 / \sigma$ which tends to 2 as $N \rightarrow \infty$.

The idea of the QP interpolation is introduced in $[1,2]$ where it is investigated based on the results of numerical experiments. Explicit representation of the interpolation is derived in [3-5]. There, the convergence of the interpolation is considered in the framework of the $L_{2}(-1,1)$-norm and at the endpoints $x= \pm 1$ in terms of the limit function. Pointwise convergence in the interval $(-1,1)$ is explored in [6]. The main results there, which we need for further comparison, are the following theorems.
Let

$$
\begin{gathered}
A_{s k}(f)=f^{(k)}(1)-(-1)^{k+s} f^{(k)}(-1), \quad k=0, \ldots, q, \\
\Phi_{k, m}\left(e^{i \pi x}\right)=e^{(i \pi / 2)(m-1) x} \sum_{r=-\infty}^{\infty} \frac{(-1)^{r(m+1)}}{(2 r+x)^{k+1}} .
\end{gathered}
$$

We denote by $R_{N, m}(f, x)$ the error of the QP interpolation as follows:

$$
R_{N, m}(f, x)=f(x)-I_{N, m}(f, x) .
$$

Theorem 1 (see [6]). Let $f^{(q+2 m)} \in A C[-1,1]$ for some $m \geq 1$, $q \geq 0$, and

$$
f^{(k)}(-1)=f^{(k)}(1)=0, \quad k=0, \ldots, q-1 .
$$


Then, the following estimate holds for $|x|<1$ as $N \rightarrow \infty$ :

$$
\begin{aligned}
R_{N, m}(f, x)= & C_{q, m}(f) \frac{(-1)^{N}}{N^{q+m+1}} \\
\times & \sin (\pi(N+1) \sigma x) \sum_{k=0}^{[m / 2]}\left(\begin{array}{c}
m-k \\
k
\end{array}\right) \\
& \times \frac{(-1)^{k}}{2^{2 k+1} \cos ^{2 k+2}(\pi x / 2)}-\sin (\pi N \sigma x) \\
& \times \sum_{k=0}^{[m / 2]-1}\left(\begin{array}{c}
m-k-2 \\
k
\end{array}\right) \\
& \left.\times \frac{(-1)^{k}}{2^{2 k+3} \cos ^{2 k+4}(\pi x / 2)}\right]+o\left(N^{-q-m-1}\right),
\end{aligned}
$$

where

$$
C_{q, m}(f)=\sum_{k=0}^{q} \frac{A_{k q}(f)(m+1)^{q-k}}{2^{q-k+1} i^{k-1} \pi^{k-m+1}(q-k) !} \Phi_{k, m}^{(m)}(-1)
$$

Theorem 2 (see [6]). Let $f^{(q+1)} \in A C[-1,1]$ for some $q \geq 0$ and

$$
f^{(k)}(-1)=f^{(k)}(1)=0, \quad k=0, \ldots, q-1 .
$$

Then, the following estimate holds for $|x|<1$ as $N \rightarrow \infty$ :

$$
\begin{aligned}
R_{N, 0}(f, x)= & A_{0 q}(f) \frac{(-1)^{N}}{2^{q+1} N^{q+1}} \frac{\sin (\pi N x)}{\cos (\pi x / 2)} \\
& \times \sum_{k=0}^{[q / 2]} \frac{(-1)^{k} 2^{2 k}}{(q-2 k) ! \pi^{2 k+1}} \sum_{s=-\infty}^{\infty} \frac{(-1)^{s}}{(2 s+1)^{2 k+1}} \\
& +o\left(N^{-q-1}\right) .
\end{aligned}
$$

In the current paper, we consider convergence acceleration of the QP interpolation by rational corrections in terms of $e^{i \pi \sigma x}$ which leads to quasi-periodic-rational (QPR) interpolation. We investigate the pointwise convergence of the QPR interpolation in the interval $(-1,1)$ and derive the exact constants of the main terms of asymptotic errors. Comparison with Theorems 1 and 2 shows the accelerated convergence for smooth functions. Some results of this research are reported also in [7].

More specifically, the QP interpolation can be realized by the following formula:

$$
I_{N, m}(f, x)=\sum_{n=-N}^{N} F_{n, m} e^{i \pi n \sigma x}, \quad m \in Z, m \geq 0,
$$

where

$$
\begin{gathered}
F_{n, m}=\check{f}_{n, m}-\sum_{\ell=1}^{m} \theta_{n, \ell} \check{f}_{\ell+N, m}, \\
\check{f}_{n, m}=\frac{1}{2 N+m+1} \sum_{k=-N}^{N} f\left(\frac{k}{N}\right) e^{-2 i \pi n k /(2 N+m+1)}, \\
\theta_{n, \ell}=e^{2 i \pi(\ell+N-n)(N+m) /(2 N+m+1)} \sum_{s=1}^{m} v_{\ell, s}^{-1} e^{2 i \pi n(s-1) /(2 N+m+1)} .
\end{gathered}
$$

Here, $v_{\ell, s}^{-1}$ are the elements of the inverse of the Vandermonde matrix $\left(v_{s, \ell}\right)$ as

$$
v_{s, \ell}=\alpha_{\ell}^{s-1}, \quad \alpha_{\ell}=e^{2 i \pi(\ell+N) /(2 N+m+1)},
$$

and have the following explicit form [8]:

$$
v_{\ell, s}^{-1}=-\frac{1}{\alpha_{\ell}^{s} \prod_{i=1, i \neq \ell}^{m}\left(\alpha_{\ell}-\alpha_{i}\right)} \sum_{j=0}^{s-1} \beta_{j} \alpha_{\ell}^{j}, \quad \ell, s=1, \ldots, m,
$$

where $\beta_{j}$ are the coefficients of the following polynomial:

$$
\prod_{j=1}^{m}\left(x-\alpha_{j}\right)=\sum_{j=0}^{m} \beta_{j} x^{j}
$$

Taking into account that $\alpha_{s}-\alpha_{i}=O(1 / N)$, from (13), we get

$$
\begin{aligned}
& v_{\ell, s}^{-1}=O\left(N^{m-1}\right), \quad N \longrightarrow \infty, \\
& \theta_{n, \ell}=O\left(N^{m-1}\right), \quad N \longrightarrow \infty .
\end{aligned}
$$

\section{Quasi-Periodic-Rational Interpolation}

In this section, we consider convergence acceleration of the QP interpolation by rational trigonometric corrections which leads to the QPR interpolation.

Consider a vector $\mu=\left\{\mu_{k}\right\}_{k=1}^{p}$. By $\Delta_{n}^{k}\left(\mu, c_{n}\right)$, we denote generalized finite differences defined by the following recurrent relations:

$$
\begin{gathered}
\Delta_{n}^{0}\left(\mu, c_{n}\right)=c_{n}, \\
\Delta_{n}^{k}\left(\mu, c_{n}\right)=\Delta_{n}^{k-1}\left(\mu, c_{n}\right)+\mu_{k} \Delta_{n-1}^{k-1}\left(\mu, c_{n}\right), \quad k \geq 1,
\end{gathered}
$$

for some sequence $c_{n}$. When $\mu \equiv 1$, we put

$$
\Delta_{n}^{k}\left(\mu, c_{n}\right)=\Delta_{n}^{k}\left(c_{n}\right) .
$$

It is easy to verify that

$$
\begin{aligned}
\Delta_{n}^{1}\left(\mu, c_{n}\right)= & c_{n}+\mu_{1} c_{n-1}, \\
\Delta_{n}^{2}\left(\mu, c_{n}\right)= & \Delta_{n}^{1}\left(\mu, c_{n}\right)+\mu_{2} \Delta_{n-1}^{1}\left(\mu, c_{n}\right) \\
= & c_{n}+\left(\mu_{1}+\mu_{2}\right) c_{n-1}+\mu_{1} \mu_{2} c_{n-2}, \\
\Delta_{n}^{3}\left(\mu, c_{n}\right)= & c_{n}+\left(\mu_{1}+\mu_{2}+\mu_{3}\right) c_{n-1} \\
& +\left(\mu_{1} \mu_{2}+\mu_{1} \mu_{3}+\mu_{2} \mu_{3}\right) c_{n-2}+\mu_{1} \mu_{2} \mu_{3} c_{n-3} .
\end{aligned}
$$


In general, we can prove by the mathematical induction that

$$
\Delta_{n}^{p}\left(\mu, c_{n}\right)=\sum_{s=0}^{p} \gamma_{s}(\mu) c_{n-s}
$$

where $\gamma_{s}(\mu)$ are the coefficients of the following polynomial:

$$
\sum_{s=0}^{p} \gamma_{s}(\mu) x^{s}=\prod_{s=1}^{p}\left(1+\mu_{s} x\right)
$$

Consider the following vectors: $\lambda=\left\{\lambda_{k}\right\}_{|k|=1}^{p}, \lambda^{+}=$ $\left\{\lambda_{k}\right\}_{k=1}^{p}$, and $\lambda^{-}=\left\{\lambda_{-k}\right\}_{k=1}^{p}$. By $\delta_{n}^{k}\left(\lambda, c_{n}\right)$, we denote modified finite differences defined by the following recurrent relations:

$$
\begin{gathered}
\delta_{n}^{0}\left(\lambda, c_{n}\right)=c_{n}, \\
\delta_{n}^{k}\left(\lambda, c_{n}\right)=\delta_{n}^{k-1}\left(\lambda, c_{n}\right)+\lambda_{-k} \delta_{n-1}^{k-1}\left(\lambda, c_{n}\right) \\
\quad+\lambda_{k}\left(\delta_{n+1}^{k-1}\left(\lambda, c_{n}\right)+\lambda_{-k} \delta_{n}^{k-1}\left(\lambda, c_{n}\right)\right), \quad k \geq 1,
\end{gathered}
$$

for some sequence $c_{n}$. When $\lambda \equiv 1$, we put

$$
\delta_{n}^{k}\left(\lambda, c_{n}\right)=\delta_{n}^{k}\left(c_{n}\right)
$$

Similar to (20), we can show that

$$
\delta_{n}^{p}\left(\lambda, c_{n}\right)=\sum_{k=0}^{p} \gamma_{k}\left(\lambda^{+}\right) \sum_{s=0}^{p} \gamma_{s}\left(\lambda^{-}\right) c_{n+k-s}
$$

where

$$
\begin{aligned}
& \prod_{k=1}^{p}\left(1+\lambda_{k} x\right)=\sum_{k=0}^{p} \gamma_{k}\left(\lambda^{+}\right) x^{k}, \\
& \prod_{k=1}^{p}\left(1+\lambda_{-k} x\right)=\sum_{k=0}^{p} \gamma_{k}\left(\lambda^{-}\right) x^{k} .
\end{aligned}
$$

It is easy to verify that

$$
\begin{gathered}
\delta_{n}^{k}\left(c_{n}\right)=\Delta_{n+k}^{2 k}\left(c_{n}\right), \\
\Delta_{n}^{k}\left(c_{n}\right)=\sum_{s=0}^{k}\left(\begin{array}{c}
k \\
s
\end{array}\right) c_{n-s}, \\
\delta_{n}^{k}\left(c_{n}\right)=\sum_{s=0}^{2 k}\left(\begin{array}{c}
2 k \\
s
\end{array}\right) c_{n+k-s} .
\end{gathered}
$$

We assume that $f \in C^{\alpha}[-1,1]$ for some $\alpha \geq 1$ and we denote

$$
f^{*}(x)= \begin{cases}f_{\text {left }}(x), & x \in[-1,-\sigma), \\ f\left(\frac{x}{\sigma}\right), & x \in[-\sigma, \sigma], \\ f_{\text {right }}(x), & x \in(\sigma, 1],\end{cases}
$$

where

$$
\begin{aligned}
& f_{\text {left }}(x)=\sum_{j=0}^{\alpha} \frac{f^{(j)}(-1)}{j !}\left(\frac{x}{\sigma}+1\right)^{j}, \\
& f_{\text {right }}(x)=\sum_{j=0}^{\alpha} \frac{f^{(j)}(1)}{j !}\left(\frac{x}{\sigma}-1\right)^{j} .
\end{aligned}
$$

According to definition of $f^{*}$, we can write

$$
f^{*}(x)=\sum_{n=-\infty}^{\infty} f_{n}^{*} e^{i \pi n x}, \quad x \in(-1,1) .
$$

Hence,

$$
f(x)=\sum_{n=-\infty}^{\infty} f_{n}^{*} e^{i \pi n \sigma x}, \quad x \in[-1,1] .
$$

Therefore,

$$
R_{N, m}(f, x)=\sum_{n=-N}^{N}\left(f_{n}^{*}-F_{n, m}\right) e^{i \pi n \sigma x}+\sum_{|n|>N} f_{n}^{*} e^{i \pi n \sigma x} .
$$

The following transformation is easy to verify (see details in [9] for similar transformation):

$$
\begin{aligned}
R_{N, m}(f, x)= & \lambda_{-1} \frac{F_{-N-1, m} e^{-i \pi \sigma N x}-F_{N, m} e^{i \pi \sigma(N+1) x}}{\left(1+\lambda_{-1} e^{i \pi \sigma x}\right)\left(1+\lambda_{1} e^{-i \pi \sigma x}\right)} \\
& +\lambda_{1} \frac{F_{N+1, m} e^{i \pi \sigma N x}-F_{-N, m} e^{-i \pi \sigma(N+1) x}}{\left(1+\lambda_{-1} e^{i \pi \sigma x}\right)\left(1+\lambda_{1} e^{-i \pi \sigma x}\right)} \\
& +\frac{1}{\left(1+\lambda_{-1} e^{i \pi \sigma x}\right)\left(1+\lambda_{1} e^{-i \pi \sigma x}\right)} \\
& \times \sum_{n=-N}^{N} \delta_{n}^{1}\left(\lambda, f_{n}^{*}-F_{n, m}\right) e^{i \pi n \sigma x} \\
& +\frac{1}{\left(1+\lambda_{-1} e^{i \pi \sigma x}\right)\left(1+\lambda_{1} e^{-i \pi \sigma x}\right)} \\
& \times \sum_{|n|>N} \delta_{n}^{1}\left(\lambda, f_{n}^{*}\right) e^{i \pi n \sigma x} .
\end{aligned}
$$

Reiteration of it up to $p$ times leads to the following expansion of the error:

$$
\begin{aligned}
R_{N, m} & (f, x) \\
& =\sum_{k=1}^{p} \lambda_{-k} \frac{\delta_{-N-1}^{k-1}\left(\lambda, F_{n, m}\right) e^{-i \pi \sigma N x}-\delta_{N}^{k-1}\left(\lambda, F_{n, m}\right) e^{i \pi \sigma(N+1) x}}{\prod_{s=1}^{k}\left(1+\lambda_{-s} e^{i \pi \sigma x}\right)\left(1+\lambda_{s} e^{-i \pi \sigma x}\right)}
\end{aligned}
$$




$$
\begin{aligned}
& +\sum_{k=1}^{p} \lambda_{k} \frac{\delta_{N+1}^{k-1}\left(\lambda, F_{n, m}\right) e^{i \pi \sigma N x}-\delta_{-N}^{k-1}\left(\lambda, F_{n, m}\right) e^{-i \pi \sigma(N+1) x}}{\prod_{s=1}^{k}\left(1+\lambda_{-s} e^{i \pi \sigma x}\right)\left(1+\lambda_{s} e^{-i \pi \sigma x}\right)} \\
& +\frac{1}{\prod_{s=1}^{p}\left(1+\lambda_{-s} e^{i \pi \sigma x}\right)\left(1+\lambda_{s} e^{-i \pi \sigma x}\right)} \\
& \times \sum_{|n| \leq N} \delta_{n}^{p}\left(\lambda, f_{n}^{*}-F_{n, m}\right) e^{i \pi n \sigma x} \\
& +\frac{1}{\prod_{s=1}^{p}\left(1+\lambda_{-s} e^{i \pi \sigma x}\right)\left(1+\lambda_{s} e^{-i \pi \sigma x}\right)} \\
& \times \sum_{|n|>N} \delta_{n}^{p}\left(\lambda, f_{n}^{*}\right) e^{i \pi n \sigma x},
\end{aligned}
$$

where the first two terms can be assumed as corrections of the error. This observation leads to the following QPR interpolation:

$$
\begin{aligned}
& I_{N, m}^{p}(f, x) \\
& \quad=I_{N, m}(f, x) \\
& \quad+\sum_{k=1}^{p} \lambda_{-k} \frac{\delta_{-N-1}^{k-1}\left(\lambda, F_{n, m}\right) e^{-i \pi \sigma N x}-\delta_{N}^{k-1}\left(\lambda, F_{n, m}\right) e^{i \pi \sigma(N+1) x}}{\prod_{s=1}^{k}\left(1+\lambda_{-s} e^{i \pi \sigma x}\right)\left(1+\lambda_{s} e^{-i \pi \sigma x}\right)} \\
& +\sum_{k=1}^{p} \lambda_{k} \frac{\delta_{N+1}^{k-1}\left(\lambda, F_{n, m}\right) e^{i \pi \sigma N x}-\delta_{-N}^{k-1}\left(\lambda, F_{n, m}\right) e^{-i \pi \sigma(N+1) x}}{\prod_{s=1}^{k}\left(1+\lambda_{-s} e^{i \pi \sigma x}\right)\left(1+\lambda_{s} e^{-i \pi \sigma x}\right)}
\end{aligned}
$$

with the error

$$
\begin{aligned}
R_{N, m}^{p}(f, x)= & \frac{1}{\prod_{s=1}^{p}\left(1+\lambda_{-s} e^{i \pi \sigma x}\right)\left(1+\lambda_{s} e^{-i \pi \sigma x}\right)} \\
& \times \sum_{|n| \leq N} \delta_{n}^{p}\left(\lambda, f_{n}^{*}-F_{n, m}\right) e^{i \pi \sigma n x} \\
& +\frac{1}{\prod_{s=1}^{p}\left(1+\lambda_{-s} e^{i \pi \sigma x}\right)\left(1+\lambda_{s} e^{-i \pi \sigma x}\right)} \\
& \times \sum_{|n|>N} \delta_{n}^{p}\left(\lambda, f_{n}^{*}\right) e^{i \pi \sigma n x} .
\end{aligned}
$$

The QPR interpolation is undefined until parameters $\lambda_{k}$ are unknown. Hence, determination of these parameters is a crucial problem for realization of the QPR interpolation. First, we assume that

$$
\lambda_{-k}=\lambda_{k}=1-\frac{\tau_{k}}{N}, \quad k=1, \ldots, p
$$

where $\tau_{k}$ are some new parameters independent of $N$. In the next section, we investigate convergence of the QPR interpolation independent of the choice of parameters $\tau_{k}$. Then, we discuss some choices of these parameters. We also consider an approach connected with the idea of the FourierPade interpolation which leads to quasi-periodic FourierPade interpolation.

\section{Convergence Analysis}

Let $\lambda_{k}$ be chosen as in (38) and let $\gamma_{k}(\tau)$ be the coefficients of the following polynomial:

$$
\prod_{s=1}^{p}\left(1+\tau_{s} x\right)=\sum_{k=0}^{p} \gamma_{k}(\tau) x^{k}
$$

where $\tau=\left\{\tau_{1}, \ldots, \tau_{p}\right\}$.

Let us modify (20) in view of (38). For $p=1$, we write

$$
\Delta_{n}^{1}\left(\lambda^{+}, c_{n}\right)=c_{n}+\left(1-\frac{\tau_{1}}{N}\right) c_{n-1}=\Delta_{n}^{1}\left(c_{n}\right)-\frac{\tau_{1}}{N} c_{n-1} .
$$

For $p=2$, we have

$$
\begin{aligned}
\Delta_{n}^{2}\left(\lambda^{+}, c_{n}\right)= & \Delta_{n}^{1}\left(\lambda^{+}, c_{n}\right)+\left(1-\frac{\tau_{2}}{N}\right) \Delta_{n-1}^{1}\left(\lambda^{+}, c_{n}\right) \\
= & \Delta_{n}^{1}\left(c_{n}\right)-\frac{\tau_{1}}{N} c_{n-1} \\
& +\left(1-\frac{\tau_{2}}{N}\right)\left(\Delta_{n-1}^{1}\left(c_{n}\right)-\frac{\tau_{1}}{N} c_{n-2}\right) \\
= & \Delta_{n}^{2}\left(c_{n}\right)-\frac{\tau_{1}+\tau_{2}}{N} \Delta_{n-1}^{1}\left(c_{n}\right)+\frac{\tau_{1} \tau_{2}}{N^{2}} \Delta_{n-2}^{0}\left(c_{n}\right) .
\end{aligned}
$$

In general, we can prove by the mathematical induction the following expansion [10]:

$$
\Delta_{n}^{p}\left(\lambda^{+}, c_{n}\right)=\sum_{s=0}^{p} \gamma_{s}\left(\lambda^{+}\right) c_{n-s}=\sum_{s=0}^{p}(-1)^{s} \frac{\gamma_{s}(\tau)}{N^{s}} \Delta_{n-s}^{p-s}\left(c_{n}\right) .
$$

Now, let us modify (24) in view of (38). According to (20) and (42), we get (note that $\lambda^{+}=\lambda^{-}$)

$$
\begin{aligned}
\delta_{n}^{p}\left(\lambda, c_{n}\right) & =\sum_{k=0}^{p} \gamma_{k}\left(\lambda^{+}\right) \sum_{s=0}^{p} \gamma_{s}\left(\lambda^{-}\right) c_{n+k-s} \\
& =\sum_{s=0}^{p}(-1)^{s} \frac{\gamma_{s}(\tau)}{N^{s}} \sum_{k=0}^{p} \gamma_{k}\left(\lambda^{+}\right) \Delta_{n+k-s}^{p-s}\left(c_{n}\right) .
\end{aligned}
$$

Similar to (42), we can show that

$$
\sum_{k=0}^{p} \gamma_{k}\left(\lambda^{+}\right) c_{n+k}=\sum_{k=0}^{p}(-1)^{k} \frac{\gamma_{k}(\tau)}{N^{k}} \Delta_{n+p}^{p-k}\left(c_{n}\right)
$$

Then, from (43), we have

$$
\delta_{n}^{p}\left(\lambda, c_{n}\right)=\sum_{s=0}^{p}(-1)^{s} \frac{\gamma_{s}(\tau)}{N^{s}} \sum_{k=0}^{p}(-1)^{k} \frac{\gamma_{k}(\tau)}{N^{k}} \Delta_{n+p}^{p-k}\left(\Delta_{n-s}^{p-s}\left(c_{n}\right)\right) .
$$

This leads to the following needed expansion:

$$
\delta_{n}^{p}\left(\lambda, c_{n}\right)=\sum_{s=0}^{p}(-1)^{s} \frac{\gamma_{s}(\tau)}{N^{s}} \sum_{k=0}^{p}(-1)^{k} \frac{\gamma_{k}(\tau)}{N^{k}} \Delta_{n+p-s}^{2 p-k-s}\left(c_{n}\right)
$$

as $\Delta_{n+p}^{p-k}\left(\Delta_{n-s}^{p-s}\left(c_{n}\right)\right)=\Delta_{n+p-s}^{2 p-k-s}\left(c_{n}\right)$. 
Then,

$$
\begin{aligned}
\delta_{n}^{w} & \left(\delta_{n}^{p}\left(\lambda, c_{n}\right)\right) \\
& =\sum_{s=0}^{p}(-1)^{s} \frac{\gamma_{s}(\tau)}{N^{s}} \sum_{k=0}^{p}(-1)^{k} \frac{\gamma_{k}(\tau)}{N^{k}} \Delta_{n+w}^{2 w}\left(\Delta_{n+p-s}^{2 p-k-s}\left(c_{n}\right)\right) .
\end{aligned}
$$

Taking into account that

$$
\Delta_{n+w}^{2 w}\left(\Delta_{n+p-s}^{2 p-k-s}\left(c_{n}\right)\right)=\Delta_{n+p-s+w}^{2 w+2 p-k-s}\left(c_{n}\right)
$$

we find that

$$
\begin{aligned}
\delta_{n}^{w} & \left(\delta_{n}^{p}\left(\lambda, c_{n}\right)\right) \\
& =\sum_{s=0}^{p}(-1)^{s} \frac{\gamma_{s}(\tau)}{N^{s}} \sum_{k=0}^{p}(-1)^{k} \frac{\gamma_{k}(\tau)}{N^{k}} \Delta_{n+p-s+w}^{2 w+2 p-k-s}\left(c_{n}\right) .
\end{aligned}
$$

We will frequently use the latest formula.

We denote by $f_{n}$ the $n$th Fourier coefficient of $f$ as

$$
f_{n}=\frac{1}{2} \int_{-1}^{1} f(x) e^{-i \pi n x} d x .
$$

Let

$$
\begin{aligned}
B_{n}(j) & =\frac{(-1)^{n+1}}{2(i \pi n)^{j+1}}, \quad n \neq 0, B_{0}(j)=0, \\
\psi_{p, m, j}^{ \pm}(\tau)= & \sum_{s=0}^{p}(-1)^{s} \gamma_{s}(\tau) \sum_{k=0}^{p} \gamma_{k}(\tau)(2 p-k-s+j) ! \\
& \times \sum_{r=-\infty}^{\infty} \frac{(-1)^{r(m+1)}}{(2 r \pm 1)^{2 p-k-s+j+1}} .
\end{aligned}
$$

First, we prove some lemmas.

Lemma 3. Let $f^{(q+2 p+m)} \in A C[-1,1]$ for some $q \geq 0, p, m \geq$ 1 , and

$$
f^{(k)}(-1)=f^{(k)}(1)=0, \quad k=0, \ldots, q-1 .
$$

Let parameters $\lambda_{k}$ be chosen as in (38). Then,

$$
\begin{array}{r}
\delta_{n}^{w}\left(\delta_{n}^{p}\left(\lambda, f_{n}^{*}\right)\right)=O\left(n^{-2 w-1} N^{-2 p-q}\right)+o\left(n^{-q-2 p-m-1}\right), \\
|n|>N, N \longrightarrow \infty .
\end{array}
$$

Proof. We have (see details in [5])

$$
\begin{aligned}
f_{n}^{*}= & \sum_{j=q}^{q+2 p+m} \frac{1}{2^{j} N^{j}} \sum_{k=0}^{j} \frac{A_{k j}(f)(m+1)^{j-k}(2 N+m+1)^{k}}{(j-k) !} B_{n}(k) \\
& +o\left(n^{-q-2 p-m-1}\right),
\end{aligned}
$$

and, consequently,

$$
\begin{aligned}
\delta_{n}^{w}\left(\delta_{n}^{p}\left(\lambda, f_{n}^{*}\right)\right)= & \sum_{j=q}^{q+2 p+m} \frac{1}{2^{j} N^{j}} \\
& \times \sum_{k=0}^{j} \frac{A_{k j}(f)(m+1)^{j-k}(2 N+m+1)^{k}}{(j-k) !} \\
& \times \delta_{n}^{w}\left(\delta_{n}^{p}\left(\lambda, B_{n}(k)\right)\right)+o\left(n^{-q-2 p-m-1}\right) .
\end{aligned}
$$

Taking into account (49) and estimating $\Delta_{n}^{p}\left(B_{n}(k)\right)=$ $O\left(n^{-k-1-p}\right)$ (see details in [9]), we get

$$
\delta_{n}^{w}\left(\delta_{n}^{p}\left(\lambda, B_{n}(k)\right)\right)=O\left(n^{-2 w-k-1} N^{-2 p}\right)
$$

which completes the proof.

Lemma 4. Let $\lambda_{k}$ be chosen as in (38), and let $\beta \in \mathbb{R}$ be a constant. Then, the following estimate holds for $n \in \mathbb{Z}$ as $N \rightarrow$ $\infty$ :

$$
\begin{aligned}
\delta_{n}^{w}\left(\delta_{n}^{p}\left(\lambda,(-1)^{n} e^{i \pi \beta n /(2 N+m+1)}\right)\right) \\
=\left(\frac{\pi \beta}{2}\right)^{2 w} \frac{(-1)^{n} e^{i \pi \beta n /(2 N+m+1)}}{N^{2 w+2 p}} h_{p}(\beta, \tau) \\
\quad+O\left(N^{-2 w-2 p-1}\right),
\end{aligned}
$$

where

$$
h_{p}(\beta, \tau)=\left(\frac{\pi \beta}{2}\right)^{2 p} \sum_{s=0}^{p} \gamma_{s}(\tau) \sum_{k=0}^{p}(-1)^{k} \gamma_{k}(\tau)\left(\frac{2}{i \pi \beta}\right)^{k+s} .
$$

Proof. From (49), we have

$$
\begin{aligned}
\delta_{n}^{w} & \left(\delta_{n}^{p}\left(\lambda,(-1)^{n} e^{i \pi \beta n /(2 N+m+1)}\right)\right) \\
= & \sum_{s=0}^{p}(-1)^{s} \frac{\gamma_{s}(\tau)}{N^{s}} \sum_{k=0}^{p}(-1)^{k} \frac{\gamma_{k}(\tau)}{N^{k}} \\
& \times \Delta_{n+p-s+w}^{2 w+2 p-k-s}\left((-1)^{n} e^{i \pi \beta n /(2 N+m+1)}\right) .
\end{aligned}
$$

In view of (27), we write

$$
\begin{aligned}
& \Delta_{n}^{p}\left((-1)^{n} e^{i \pi \beta n /(2 N+m+1)}\right) \\
&=(-1)^{n} \sum_{k=0}^{p}\left(\begin{array}{l}
p \\
k
\end{array}\right)(-1)^{k} e^{i \pi \beta(n-k) /(2 N+m+1)} \\
&=(-1)^{n} e^{i \pi \beta n /(2 N+m+1)} \sum_{k=0}^{p}\left(\begin{array}{l}
p \\
k
\end{array}\right)(-1)^{k} \\
& \times \sum_{t=0}^{\infty} \frac{(i \pi \beta)^{t}(-1)^{t} k^{t}}{t !(2 N+m+1)^{t}} \\
&=(-1)^{n} e^{i \pi \beta n /(2 N+m+1)} \sum_{t=0}^{\infty} \frac{(-1)^{t}(i \pi \beta)^{t}}{t !(2 N+m+1)^{t}} \omega_{p, t},
\end{aligned}
$$


where

$$
\omega_{p, t}=\sum_{s=0}^{p}\left(\begin{array}{l}
p \\
s
\end{array}\right)(-1)^{s} s^{t} .
$$

Taking into account that $\omega_{p, j}=0,0 \leq j<p$ and $\omega_{p, p}=$ $(-1)^{p} p$ ! (see [10], Lemma 2.1), we get

$$
\begin{aligned}
\Delta_{n}^{p} & \left((-1)^{n} e^{i \pi \beta n /(2 N+m+1)}\right) \\
& =\frac{(-1)^{n}(i \pi \beta)^{p}}{2^{p} N^{p}} e^{i \pi \beta n /(2 N+m+1)}+O\left(N^{-p-1}\right) .
\end{aligned}
$$

Then,

$$
\begin{aligned}
\Delta_{n+p-s+w}^{2 w+2 p-k-s}\left((-1)^{n} e^{i \pi \beta n /(2 N+m+1)}\right) \\
=(-1)^{n+p+s+w}\left(\frac{i \pi \beta}{2 N}\right)^{2 w+2 p-k-s} e^{i \pi \beta(n+p-s+w) /(2 N+m+1)} \\
\quad+O\left(N^{-2 w-2 p+k+s-1}\right) \\
=(-1)^{n+p+s+w} e^{i \pi \beta n /(2 N+m+1)}\left(\frac{i \pi \beta}{2 N}\right)^{2 w+2 p-k-s} \\
\quad+O\left(N^{-2 w-2 p+k+s-1}\right)
\end{aligned}
$$

which completes the proof together with (59).

Lemma 5. Let $f^{(q+2 p+m)} \in A C[-1,1]$ for some $q \geq 0, p, m \geq$ 1 , and

$$
f^{(k)}(-1)=f^{(k)}(1)=0, \quad k=0, \ldots, q-1 .
$$

Let parameters $\lambda_{k}$ be chosen as in (38). Then, the following estimate holds as $N \rightarrow \infty$ :

$$
\begin{array}{r}
\delta_{n}^{w}\left(\delta_{n}^{p}\left(\lambda, F_{n, m}-f_{n}^{*}\right)\right)=O\left(N^{-q-2 w-2 p-1}\right)+o\left(N^{-q-2 p-2}\right), \\
-N \leq n \leq N+m .
\end{array}
$$

Proof. First, we estimate $F_{n, m}-f_{n}^{*}$. We have (see details in [5], at the beginning of the proof of Lemma 5)

$$
\begin{gathered}
F_{n, m}=\sum_{r=-\infty}^{\infty} f_{n+r(2 N+m+1)}^{*}-\sum_{\ell=1}^{m} \theta_{n, \ell} \sum_{r=-\infty}^{\infty} f_{N+\ell+r(2 N+m+1)}^{*}, \\
F_{n, m}-f_{n}^{*}=\sum_{r \neq 0} f_{n+r(2 N+m+1)}^{*}-\sum_{\ell=1}^{m} \theta_{n, \ell} \sum_{r=-\infty}^{\infty} f_{N+\ell+r(2 N+m+1)}^{*} .
\end{gathered}
$$

Then, in view of (54), we get

$$
\begin{aligned}
& \sum_{r \neq 0} f_{n+r(2 N+m+1)}^{*} \\
& =\sum_{j=q}^{q+2 p+m} \frac{1}{2^{j} N^{j}} \\
& \times \sum_{k=0}^{j} \frac{A_{k j}(f)(m+1)^{j-k}(2 N+m+1)^{k}}{(j-k) !} \\
& \times \sum_{r \neq 0} B_{n+r(2 N+m+1)}(k)+o\left(N^{-q-2 p-m-1}\right), \\
& \sum_{\ell=1}^{m} \theta_{n, \ell} \sum_{r=-\infty}^{\infty} f_{N+\ell+r(2 N+m+1)}^{*} \\
& =\frac{(-1)^{n+1}}{2 N+m+1} \\
& \times \sum_{j=q}^{q+2 p+m} \frac{1}{N^{j}} \sum_{k=0}^{j} \frac{A_{k j}(f)(m+1)^{j-k}}{2^{j-k}(i \pi)^{k+1}(j-k) !} \\
& \times e^{-i \pi(m-1) n /(2 N+m+1)} \sum_{\ell=1}^{m} \Phi_{k, m}\left(e^{2 i \pi(N+\ell) /(2 N+m+1)}\right) \\
& \times \sum_{s=1}^{m} v_{\ell, s}^{-1} e^{2 \imath \pi n(s-1) /(2 N+m+1)}+o\left(N^{-q-2 p-2}\right),
\end{aligned}
$$

where we used estimate (16).

According to the Taylor expansion

$$
\begin{aligned}
\Phi_{k, m} & \left(e^{2 \imath \pi(N+\ell) /(2 N+m+1)}\right) \\
= & \sum_{t=0}^{2 p+m} \frac{1}{t !} \Phi_{k, m}^{(t)}(-1)\left(e^{2 i \pi(N+\ell) /(2 N+m+1)}+1\right)^{t} \\
& +O\left(N^{-2 p-m-1}\right)
\end{aligned}
$$

and relations

$$
\begin{gathered}
\sum_{\ell=1}^{m}\left(e^{2 i \pi(N+\ell) /(2 N+m+1)}+1\right)^{\tau} \sum_{s=1}^{m} v_{\ell, s}^{-1} e^{2 i \pi n(s-1) /(2 N+m+1)} \\
=\left(e^{2 i \pi n /(2 N+m+1)}+1\right)^{\tau}, \quad \tau=0, \ldots, m-1,
\end{gathered}
$$

we derive

$$
\begin{aligned}
F_{n, m}-f_{n}^{*}= & \sum_{j=q}^{q+2 p+m} \frac{1}{2^{j} N^{j}} \\
& \times \sum_{k=0}^{j} \frac{A_{k j}(f)(m+1)^{j-k}(2 N+m+1)^{k}}{(j-k) !} \\
& \times \sum_{r \neq 0} B_{n+r(2 N+m+1)}(k)
\end{aligned}
$$




$$
\begin{aligned}
& +\frac{(-1)^{n} e^{-i \pi(m-1) n /(2 N+m+1)}}{2 N+m+1} \\
& \times \sum_{j=q}^{q+2 p+m} \frac{1}{N^{j}} \sum_{k=0}^{j} \frac{A_{k j}(f)(m+1)^{j-k}}{2^{j-k}(i \pi)^{k+1}(j-k) !} \\
& \times\left[\sum_{t=0}^{m-1} \frac{\Phi_{k, m}^{(t)}(-1)}{t !}\left(e^{2 i \pi n /(2 N+m+1)}+1\right)^{t}\right. \\
& +\sum_{t=m}^{m+2 p} \frac{\Phi_{k, m}^{(t)}(-1)}{t !} \\
& \times \sum_{\ell=1}^{m}\left(e^{2 i \pi(N+\ell) /(2 N+m+1)}+1\right)^{t} \\
& \left.\quad \times \sum_{s=1}^{m} v_{\ell, s}^{-1} e^{2 i \pi n(s-1) /(2 N+m+1)}\right] \\
& +o\left(N^{-q-2 p-2}\right) .
\end{aligned}
$$

This completes the proof in view of (49), Lemma 4, and the following estimate [9]:

$$
\delta_{n}^{w}\left(\delta_{n}^{p}\left(\lambda, \sum_{r \neq 0} B_{n+r(2 N+m+1)}(k)\right)\right)=O\left(N^{-2 w-2 p-k-1}\right) .
$$

We used also the fact that $e^{2 i \pi(N+\ell) /(2 N+m+1)}+1=O(1 / N)$.

Lemma 6. Let $f^{(q+2 p+m)} \in A C[-1,1]$ for some $q \geq 0, p, m \geq$ 1 , and

$$
f^{(k)}(-1)=f^{(k)}(1)=0, \quad k=0, \ldots, q-1 .
$$

Let parameters $\lambda_{k}$ be chosen as in (38). Then,

$$
\begin{aligned}
& \delta_{ \pm N}^{p}\left(\lambda, F_{n, m}\right)=\frac{(-1)^{N+1}}{2^{q+1} N^{q+2 p+1}} \\
& \times \sum_{k=0}^{q} \frac{A_{k q}(f)(m+1)^{q-k} 2^{k}}{(q-k) !(i \pi)^{k+1}} \\
& \times\left[(-1)^{p} \frac{\psi_{p, m, k}^{ \pm}(\tau)}{k !}-\sum_{t=0}^{m-1} \frac{\Phi_{k, m}^{(t)}(-1)}{t !}\right. \\
& \times \sum_{\mu=0}^{t}\left(\begin{array}{c}
t \\
\mu
\end{array}\right) e^{ \pm i \pi N(2 \mu-m+1) /(2 N+m+1)} \\
& \left.\times h_{p}(2 \mu-m+1, \tau)\right] \\
& +O\left(N^{-q-2 p-2}\right) \\
& \delta_{ \pm(N+1)}^{p}\left(\lambda, F_{n, m}\right)=-\delta_{ \pm N}^{p}\left(\lambda, F_{n, m}\right)+O\left(N^{-q-2 p-2}\right), \\
& \delta_{-N}^{p}\left(\lambda, F_{n, m}\right)=(-1)^{m+1} \delta_{N}^{p}\left(\lambda, F_{n, m}\right)+O\left(N^{-q-2 p-2}\right) .
\end{aligned}
$$

Proof. We proceed as in the proof of Lemma 5 and derive

$$
\begin{aligned}
& \delta_{ \pm N}^{p}\left(\lambda, F_{n, m}\right)=\sum_{j=q}^{q+2 p+m} \frac{1}{2^{j} N^{j}} \\
& \times \sum_{k=0}^{j} \frac{A_{k j}(f)(2 N+m+1)^{k}}{(j-k) !(m+1)^{k-j}} \\
& \times \delta_{ \pm N}^{p}\left(\lambda, \sum_{r=-\infty}^{\infty} B_{n+r(2 N+m+1)}(k)\right) \\
& +\frac{1}{2 N+m+1} \sum_{j=q}^{q+2 p+m} \frac{1}{N^{j}} \\
& \times \sum_{k=0}^{j} \frac{A_{k j}(f)(m+1)^{j-k}}{2^{j-k}(i \pi)^{k+1}(j-k) !} \\
& \times\left[\sum_{t=0}^{m-1} \frac{\Phi_{k, m}^{(t)}(-1)}{t !} \sum_{\mu=0}^{t}\left(\begin{array}{c}
t \\
\mu
\end{array}\right)\right. \\
& \times \delta_{ \pm N}^{p}\left(\lambda,(-1)^{n} e^{i \pi n(2 \mu-m+1) /(2 N+m+1)}\right) \\
& +\sum_{t=m}^{m+2 p} \frac{\Phi_{k, m}^{(t)}(-1)}{t !} \\
& \times \sum_{\ell=1}^{m}\left(e^{2 i \pi(N+\ell) /(2 N+m+1)}+1\right)^{t} \\
& \times \sum_{s=1}^{m} v_{\ell, s}^{-1} \\
& \left.\times \delta_{ \pm N}^{p}\left(\lambda,(-1)^{n} e^{i \pi n(2 s-1-m) /(2 N+m+1)}\right)\right] \\
& +o\left(N^{-q-2 p-2}\right) \text {. }
\end{aligned}
$$

This completes the proof in view of Lemma 4 and the following estimate:

$$
\begin{aligned}
\delta_{ \pm N}^{w}\left(\delta_{n}^{p}\left(\lambda, \sum_{r=-\infty}^{\infty} B_{n+r(2 N+m+1)}(j)\right)\right) \\
=\frac{(-1)^{N+p+w+1}}{2(i \pi N)^{j+1} N^{2 w+2 p} j !} \psi_{p, m, j+2 w}^{ \pm}(\tau) \\
+O\left(N^{-2 w-2 p-j-2}\right) .
\end{aligned}
$$

The proof of (77), for $m=0$, can be found in [9]. General case can be proved similarly and we omit it.

Estimates (74) and (75) can be proved similarly.

Now, we present the main results of the paper. 
Theorem 7. Let $f^{(q+2 p+m)} \in A C[-1,1]$ for some $q \geq 0, p, m \geq$ 1 , and

$$
f^{(k)}(-1)=f^{(k)}(1)=0, \quad k=0, \ldots, q-1 .
$$

Let parameters $\lambda_{k}$ be chosen as in (38). Then, the following estimate holds for $|x|<1$ :

$$
\begin{aligned}
R_{N, m}^{p}(f, x)= & \frac{(-1)^{N}}{(2 N)^{q+2 p+1}} \frac{\sin (\pi \sigma(N+(1 / 2)) x-(\pi m / 2))}{\cos ^{2 p+1}(\pi x / 2)} \\
& \times \sum_{k=0}^{q} \frac{A_{k q}(f)(m+1)^{q-k} 2^{k}}{(q-k) ! i^{k-m} \pi^{k+1}} \\
& \times\left((-1)^{p} \frac{\psi_{p, m, k}^{+}(\tau)}{k !}-\sum_{t=0}^{m-1} \frac{\Phi_{k, m}^{(t)}(-1)}{t !}\right. \\
& \left.\quad \times \sum_{\mu=0}^{t}\left(\begin{array}{c}
t \\
\mu
\end{array}\right) i^{2 \mu-m+1} h_{p}(2 \mu-m+1, \tau)\right) \\
& +o\left(N^{-q-2 p-1}\right), \quad N \longrightarrow \infty .
\end{aligned}
$$

Proof. We have from (37) by the Abel transformation (see transformation from (33) to (34) with $\lambda \equiv 1$ )

$$
\begin{aligned}
R_{N, m}^{p}(f, x)= & e^{-i \pi \sigma N x} \frac{\delta_{-N-1}^{p}\left(\lambda, F_{n, m}\right)}{c(x)} \\
& -e^{i \pi \sigma(N+1) x} \frac{\delta_{N}^{p}\left(\lambda, F_{n, m}\right)}{c(x)} \\
& +e^{i \pi \sigma N x} \frac{\delta_{N+1}^{p}\left(\lambda, F_{n, m}\right)}{c(x)} \\
& -e^{-i \pi \sigma(N+1) x} \frac{\delta_{-N}^{p}\left(\lambda, F_{n, m}\right)}{c(x)} \\
& +\frac{1}{c(x)} \sum_{n=-N}^{N} \delta_{n}^{1}\left(\delta_{n}^{p}\left(\lambda, f_{n}^{*}-F_{n, m}\right)\right) e^{i \pi \sigma n x} \\
& +\frac{1}{c(x)} \sum_{|n|>N} \delta_{n}^{1}\left(\delta_{n}^{p}\left(\lambda, f_{n}^{*}\right)\right) e^{i \pi \sigma n x},
\end{aligned}
$$

where

$$
c(x)=4 \cos ^{2}\left(\frac{\pi \sigma x}{2}\right) \prod_{s=1}^{p}\left(1+\lambda_{-s} e^{i \pi \sigma x}\right)\left(1+\lambda_{s} e^{-i \pi \sigma x}\right) .
$$

It is easy to verify that

$$
\lim _{N \rightarrow \infty} c(x)=2^{2 p+2} \cos ^{2 p+2}\left(\frac{\pi x}{2}\right) .
$$

According to Lemma 3,

$$
\begin{array}{r}
\delta_{n}^{1}\left(\delta_{n}^{p}\left(\lambda, f_{n}^{*}\right)\right)=O\left(n^{-3} N^{-2 p-q}\right)+o\left(n^{-q-2 p-2}\right), \\
|n|>N, \quad N \longrightarrow \infty .
\end{array}
$$

Hence, the last term in the right-hand side of (80) is $o\left(N^{-q-2 p-1}\right)$. Then, by Lemma 5 ,

$$
\begin{aligned}
\delta_{n}^{1}\left(\delta_{n}^{p}\left(\lambda, F_{n, m}-f_{n}^{*}\right)\right) & =O\left(N^{-q-2 p-3}\right)+o\left(N^{-q-2 p-2}\right), \\
& -N \leq n \leq N+m, \quad N \longrightarrow \infty
\end{aligned}
$$

and the fifth term is also $o\left(N^{-q-2 p-1}\right)$.

Therefore,

$$
\begin{aligned}
c(x) R_{N, m}^{p}(f, x)= & e^{-i \pi \sigma N x} \delta_{-N-1}^{p}\left(\lambda, F_{n, m}\right) \\
& -e^{i \pi \sigma(N+1) x} \delta_{N}^{p}\left(\lambda, F_{n, m}\right) \\
& +e^{i \pi \sigma N x} \delta_{N+1}^{p}\left(\lambda, F_{n, m}\right) \\
& -e^{-i \pi \sigma(N+1) x} \delta_{-N}^{p}\left(\lambda, F_{n, m}\right) \\
& +o\left(N^{-q-2 p-1}\right) .
\end{aligned}
$$

Taking into account estimates (74) and (75), we get

$$
\begin{aligned}
c(x) R_{N, m}^{p}(f, x)= & \delta_{N}^{p}\left(\lambda, F_{n, m}\right) \\
\times & \left((-1)^{m}\left(e^{-i \pi \sigma N x}+e^{-i \pi \sigma(N+1) x}\right)\right. \\
& \left.-\left(e^{i \pi \sigma N x}+e^{i \pi \sigma(N+1) x}\right)\right) \\
+ & o\left(N^{-q-2 p-1}\right)
\end{aligned}
$$

which concludes the proof in view of Lemma 6 .

Let us compare the results of Theorems 1 and 7. Theorem 1 investigates the pointwise convergence of the QP interpolation on $(-1,1)$ and states that for $f^{(q+2 m)} \in A C[-1,1]$ the convergence rate is $O\left(N^{-q-m-1}\right)$ for $m \geq 1$. Theorem 7 explores the pointwise convergence of the QPR interpolation and shows that convergence rate is $O\left(N^{-q-2 p-1}\right)$ for $f^{(q+2 p+m)} \in A C[-1,1]$ and $m \geq 1$. We see that for $m=2 p$ both theorems are provided with the same rates of convergence by putting the same smoothness requirements on $f$, although the exact constants of the asymptotic errors are different. Then, we see that for $p>m / 2$ the QPR interpolation has improved accuracy compared to the QP interpolation and improvement is by factor $O\left(N^{2 p-m}\right)$. In this case, Theorem 7 puts additional smoothness requirement on $f$ and comparison is valid if only the interpolated function has enough smoothness (for example, if it is infinitely differentiable). It is worth recalling that parameter $m$ indicates the size of the Vandermonde matrix (12) that must be inverted for realization of the QP and QPR interpolations. It is wellknown that the Vandermonde matrices are ill-conditioned and standard numerical methods fail to accurately compute the entries of the inverses when the sizes of the matrices are big. Hence, from practical point of view, it is more reasonable to take $m$ small $(m \leq 6)$ and additional accuracy obtain by increasing $p$. 


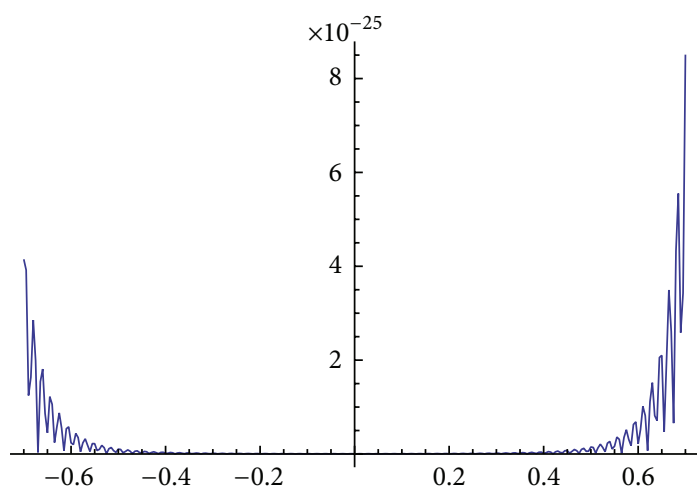

(a)

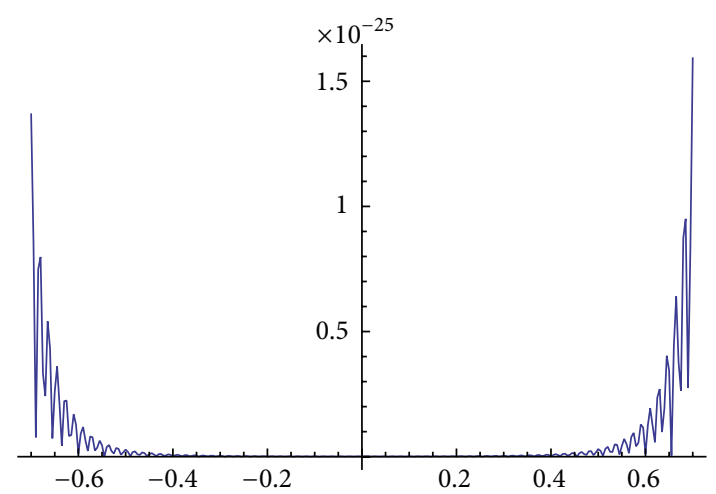

(b)

Figure 1: The graphs of the absolute errors $\left|R_{256, m}^{p}(f, x)\right|$ on interval $[-0.7,0.7]$ for $m=2$ and $p=4$ when $\tau_{k}=k$ (a) and with optimal $\tau_{k}$ (see (94)) (b) while interpolating (90).

Note that for $m=1$ the second term in the brackets of estimate (79) vanishes and also $\psi_{p, 1,0}^{+}(1, \tau)=0$. Hence,

$$
R_{N, 1}^{p}(f, x)=o\left(N^{-2 p-1}\right), \quad N \longrightarrow \infty, q=0 .
$$

Similarly, the case $m=0$ can be analyzed. We present the corresponding theorem without the proof which can be performed as the above one.

Theorem 8. Let $f^{(q+2 p+1)} \in A C[-1,1]$ for some $q \geq 0, p \geq 1$, and

$$
f^{(k)}(-1)=f^{(k)}(1)=0, \quad k=0, \ldots, q-1 .
$$

Let parameters $\lambda_{k}$ be chosen as in (38). Then, the following estimate holds for $|x|<1$ as $N \rightarrow \infty$ :

$$
\begin{aligned}
R_{N, 0}^{p}(f, x)= & \frac{(-1)^{N+p}}{(2 N)^{q+2 p+1}} \frac{\sin (\pi N x)}{\cos ^{2 p+1}(\pi x / 2)} \\
& \times \sum_{k=0}^{q} \frac{A_{k q}(f) 2^{k}}{i^{k} \pi^{k+1}(q-k) ! k !} \psi_{p, 0, k}^{+}(\tau) \\
& +o\left(N^{-q-2 p-1}\right) .
\end{aligned}
$$

Comparison with Theorem 2 shows improvement by factor $O\left(N^{2 p}\right)$ for any $p \geq 1$ if $f$ has enough smoothness.

\section{Parameter Determination in Rational Corrections}

Till now, we did not discuss the problem of parameters $\tau_{k}$ determination as Theorems 7 and 8 are valid for all choices. Now, let us consider some choices with the corresponding numerical results.

Let

$$
f(x)=\left(1-x^{2}\right)^{4} \sin (x-1) .
$$

One choice is $\tau_{k}=k$ which shows satisfactory numerical results (see Figure 1).
Another choice is based on the asymptotic estimates of Theorems 7 and 8. If it is possible to vanish the following expressions by the choice of parameters $\tau_{k}$ :

$$
\begin{array}{r}
(-1)^{p} \frac{\psi_{p, m, k}^{+}(\tau)}{k !}-\sum_{t=0}^{m-1} \frac{\Phi_{k, m}^{(t)}(-1)}{t !} \\
\times \sum_{\mu=0}^{t}\left(\begin{array}{c}
t \\
\mu
\end{array}\right) i^{2 \mu-m+1} h_{p}(2 \mu-m+1, \tau)=0, \\
k=0, \ldots, q
\end{array}
$$

or

$$
\psi_{p, 0, k}^{+}(\tau)=0, \quad k=0, \ldots, q,
$$

then, Theorems 7 and 8 will be provided with improved estimate

$$
R_{N, m}^{p}(f, x)=o\left(N^{-q-2 p-1}\right), \quad|x|<1 .
$$

For example, when $p=q=4$ and $m=2$, we find

$$
\begin{aligned}
& \tau_{1}=-0.3047909-0.4539705 i, \\
& \tau_{2}=0.9640011-0.1481304 i, \\
& \tau_{3}=6.375385+0.000739 i, \\
& \tau_{4}=12.877189-0.000022 i .
\end{aligned}
$$

With this choice $R_{256,2}^{4}(f, x)=o\left(N^{-13}\right)$ for (90).

Figure 1 compares the choices $\tau_{k}=k$ (a) and optimal choice (104) (b). As it was expected the precision of interpolation is higher in (b) compared to (a).

The third choice is not connected with (38) and allows determining parameters $\lambda_{k}$ immediately along the ideas of the Fourier-Pade interpolation ([11]). This approach is more complex as $\lambda_{k}$ must be recalculated for each function $f$ and for each $N$ and as a consequence leads to nonlinear interpolation but, however, is much more precise when $|x|<1$. 


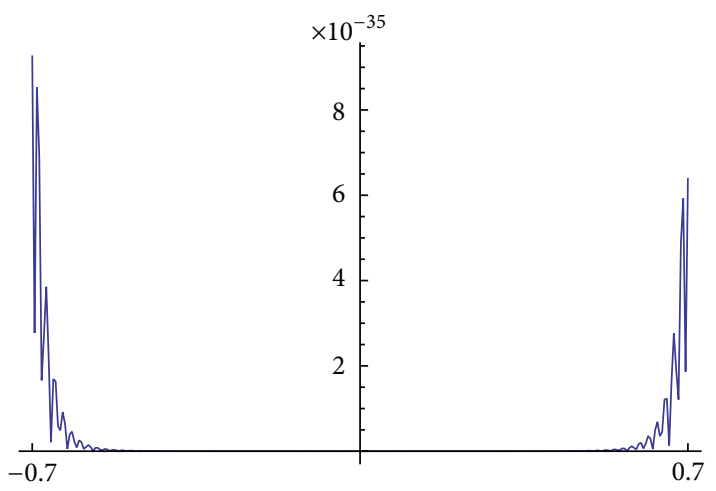

Figure 2: The graph of $\left|R_{256, m}^{p}(f, x)\right|$ on interval $[-0.7,0.7]$ for $m=2$ and $p=4$ while interpolating (90) by the quasi-periodic FourierPade interpolation (see (96)).

More specifically, parameters $\lambda$ can be determined from the following system:

$$
\delta_{n}^{p}\left(\lambda, F_{n, m}\right)=0, \quad|n|=N-p+1, \ldots, N .
$$

We will refer to this interpolation as quasi-periodic FourierPade interpolation.

For example, when $m=2, p=4$, and $N=256$, then, for (90), we get

$$
\begin{array}{ll}
\lambda_{1}=0.922+0.010 i, & \lambda_{2}=0.952+0.010 i, \\
\lambda_{3}=0.975+0.010 i, & \lambda_{4}=0.994-0.011 i, \\
\lambda_{-1}=0.994+0.011 i, & \lambda_{-2}=0.975-0.010 i, \\
\lambda_{-3}=0.952-0.010 i, & \lambda_{-4}=0.922-0.010 i .
\end{array}
$$

Figure 2 shows the graph of $\left|R_{256,2}^{4}(f, x)\right|$ for function (90) with $\lambda_{k}$ from (96). Comparison with Figure 1 shows high precision of the quasi-periodic Fourier-Pade interpolation for $|x|<1$ compared to other choices of parameters $\lambda_{k}$. Theoretical analysis of convergence of the quasi-periodic Fourier-Pade interpolation will be carried out elsewhere.

\section{Conflict of Interests}

The authors declare that there is no conflict of interests regarding the publication of this paper.

\section{Acknowledgments}

The authors acknowledge the reviewers and the editors for their valuable suggestions and constructive comments that helped to improve the paper.

\section{References}

[1] A. Nersessian and N. Hovhannisyan, "Quasiperiodic interpolation," Reports of the National Academy of Sciences of Armenia, vol. 101, no. 2, pp. 115-121, 2001.

[2] A. Nersessian and N. Hovhannisyan, "Minimization of errors of the polynomial-trigonometric interpolation with shifted nodes," 2007, Preprint of the Institute of Mathematics of the National Academy of Sciences of Armenia, http://math.sci .am/upload/preprints/toPrprt.pdf.

[3] L. Poghosyan, "On a convergence of the quasi-periodic interpolation," in Proceedings of the 2012 International Workshop on Functional Analysis, Timisoara, Romania, October 2012.

[4] L. Poghosyan, "On $\mathrm{L}_{2}$-convergence of the quasi-periodic interpolation," Reports of the National Academy of Sciences of Armenia, vol. 113, no. 3, pp. 240-247, 2013.

[5] L. Poghosyan and A. Poghosyan, "Asymptotic estimates for the quasi-periodic interpolations," Armenian Journal of Mathematics, vol. 5, no. 1, pp. 34-57, 2013.

[6] L. Poghosyan and A. Poghosyan, "On a pointwise convergence of the quasi-periodictrigonometric interpolation," Proceedings of the National Academy of Sciences of Armenia-Mathematics. In press.

[7] L. Poghosyan and A. Poghosyan, "Convergence acceleration of the quasi-periodic interpolation by rational and polynomial corrections (abstract)," in Proceedings of the 2nd International Conference Mathematics in Armenia: Advances and Perspectives, Tsaghkadzor, Armenia, August 2013.

[8] I. Gohberg and V. Olshevsky, "The fast generalized Parker-Traub algorithm for inversion of Vandermonde and related matrices," Journal of Complexity, vol. 13, no. 2, pp. 208-234, 1997.

[9] A. Poghosyan, "On a fast convergence of the rationaltrigonometric-polynomial interpolation," Advances in Numerical Analysis, vol. 2013, Article ID 315748, 13 pages, 2013.

[10] A. Nersessian and A. Poghosyan, "On a rational linear approximation of Fourier series for smooth functions," Journal of Scientific Computing, vol. 26, no. 1, pp. 111-125, 2006.

[11] G. A. Baker and P. Graves-Morris, Pade Approximants, Encyclopedia of Mathematics and Its Applications, vol. 59, Cambridge University Press, Cambridge, UK, 2nd edition, 1966. 


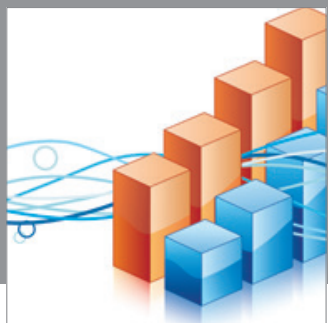

Advances in

Operations Research

mansans

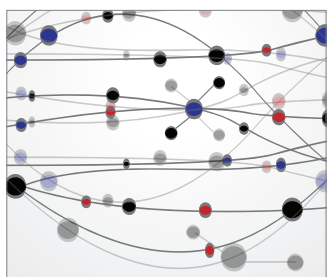

The Scientific World Journal
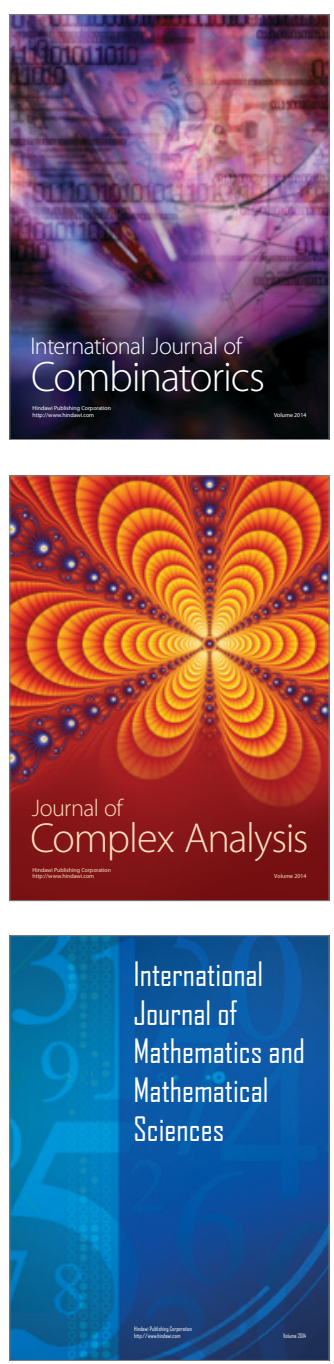
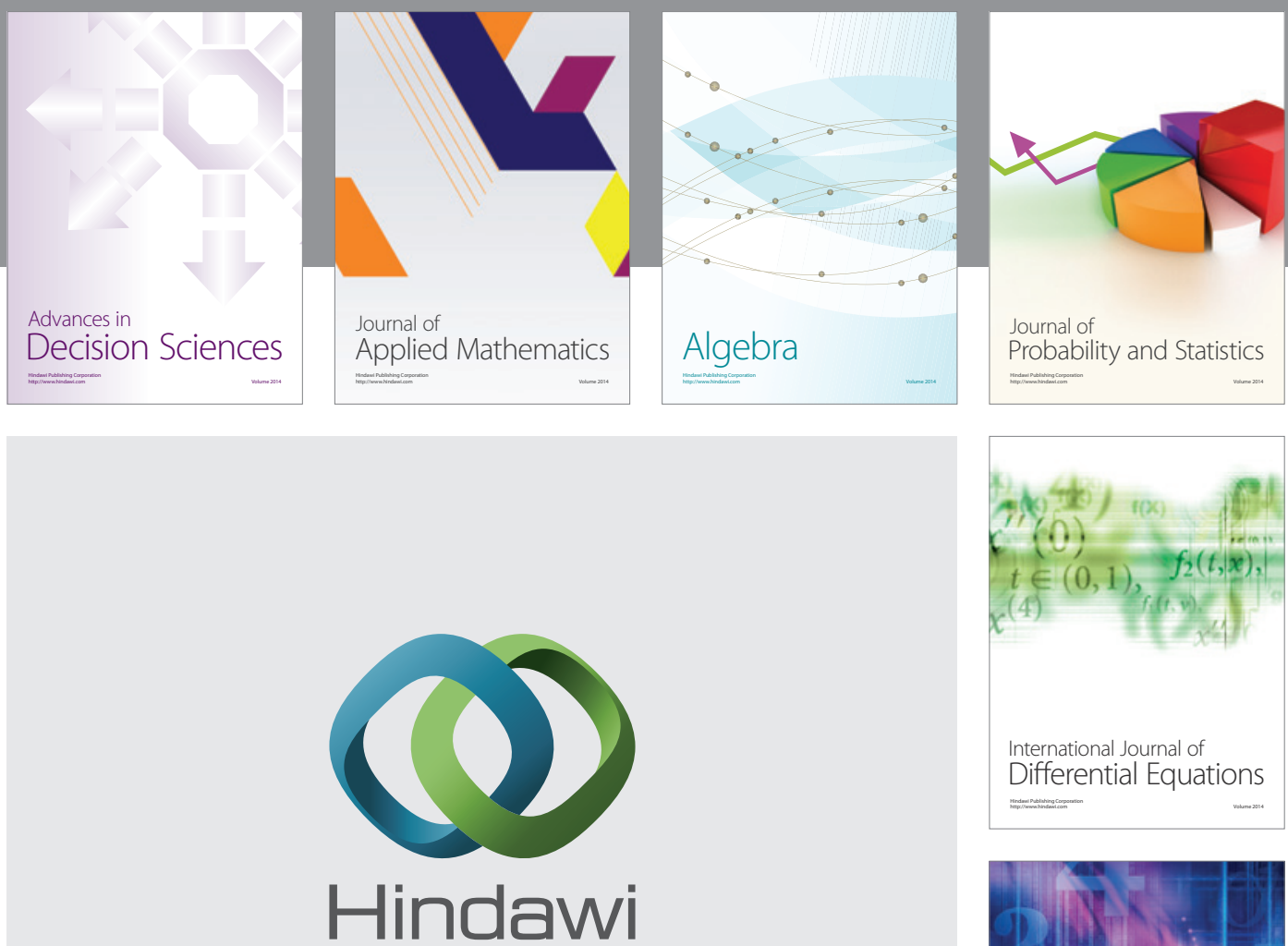

Submit your manuscripts at http://www.hindawi.com
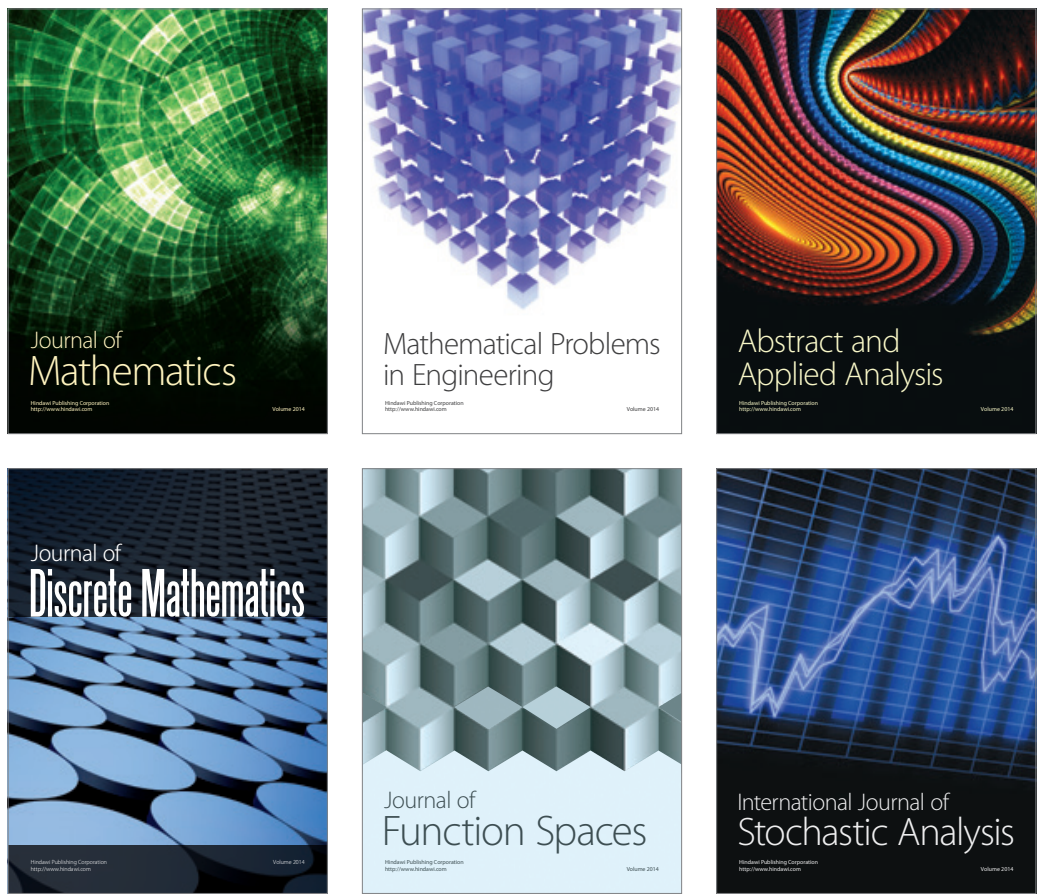

Journal of

Function Spaces

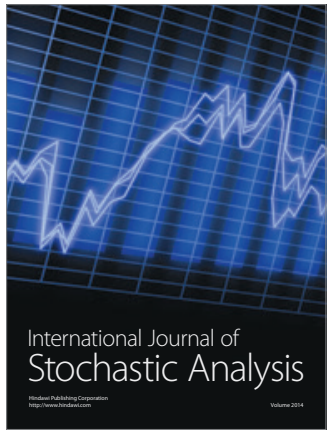

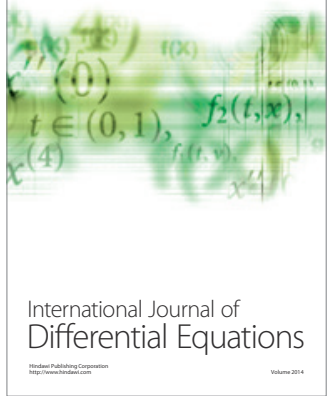
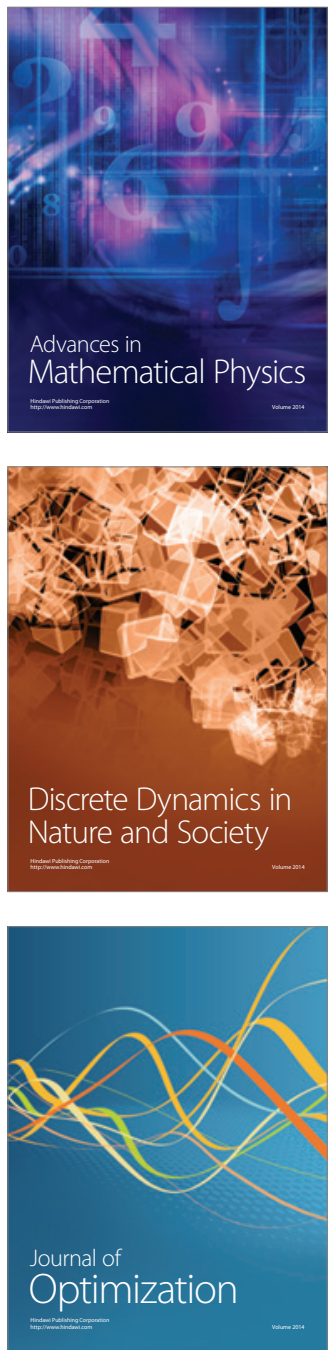